STRUCTURAL BIOLOGY

ISSN 2059-7983

Received 31 July 2019

Accepted 8 November 2019

Edited by P. Langan, Oak Ridge National

Laboratory, USA

₹ Currently at Microsoft.

Keywords: Amber refinement target; hydrogenbond quality; Amber in Phenix; $C^{\beta}$ deviations; peptide orientations.

Supporting information: this article has supporting information at journals.iucr.org/d

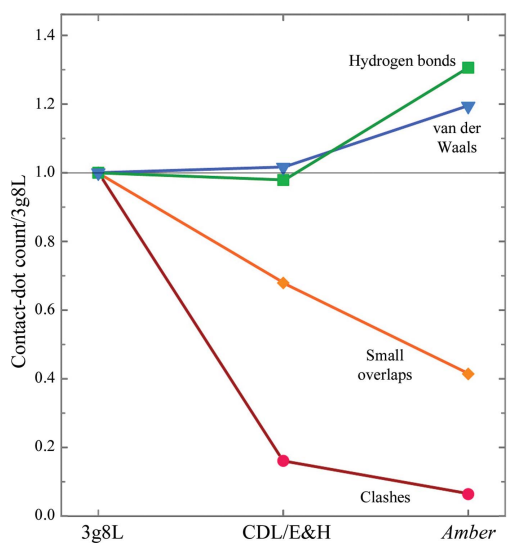

OPEN Ә ACCESS

\section{Improved chemistry restraints for crystallographic refinement by integrating the Amber force field into Phenix}

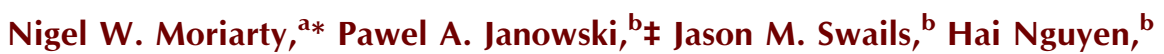 \\ Jane S. Richardson, ${ }^{c}$ David A. Case ${ }^{b}$ and Paul D. Adams ${ }^{a, d}$
}

aMolecular Biosciences and Integrated Bioimaging, Lawrence Berkeley National Laboratory, Berkeley, CA 94720-8235, USA, 'bepartment of Chemistry and Chemical Biology, Rutgers University, Piscataway, NJ 08854, USA, ' Department of Biochemistry, Duke University, Durham, NC 27710, USA, and d Department of Bioengineering, University of California at Berkeley, Berkeley, CA 94720, USA. *Correspondence e-mail: nwmoriarty@lbl.gov

The refinement of biomolecular crystallographic models relies on geometric restraints to help to address the paucity of experimental data typical in these experiments. Limitations in these restraints can degrade the quality of the resulting atomic models. Here, an integration of the full all-atom Amber molecular-dynamics force field into Phenix crystallographic refinement is presented, which enables more complete modeling of biomolecular chemistry. The advantages of the force field include a carefully derived set of torsion-angle potentials, an extensive and flexible set of atom types, Lennard-Jones treatment of nonbonded interactions and a full treatment of crystalline electrostatics. The new combined method was tested against conventional geometry restraints for over 22000 protein structures. Structures refined with the new method show substantially improved model quality. On average, Ramachandran and rotamer scores are somewhat better, clashscores and MolProbity scores are significantly improved, and the modeling of electrostatics leads to structures that exhibit more, and more correct, hydrogen bonds than those refined using traditional geometry restraints. In general it is found that model improvements are greatest at lower resolutions, prompting plans to add the Amber target function to realspace refinement for use in electron cryo-microscopy. This work opens the door to the future development of more advanced applications such as Amber-based ensemble refinement, quantum-mechanical representation of active sites and improved geometric restraints for simulated annealing.

\section{Introduction}

Accurate structural knowledge lies at the heart of our understanding of the biomolecular function and interactions of proteins and nucleic acids. With close to $90 \%$ of the structures in the Protein Data Bank (Berman et al., 2000) solved via X-ray diffraction methods, crystallography is currently the pre-eminent method for determining biomolecular structure. Crystal structure refinement is a computational technique that plays a key role in post-experiment data interpretation. The refinement of atomic coordinates entails solving an optimization problem to minimize the residual difference between the experimental and model structurefactor amplitudes (Jack \& Levitt, 1978; Agarwal, 1978; Murshudov et al., 1997). However, owing to inherent experimental limitations and a typically low data-to-parameter ratio, the employment of additional restraints, commonly referred to as geometry or steric restraints, is key to successful structural 
refinement (Waser, 1963). These restraints, which can be thought of as a prior in the Bayesian sense, provide additional observations in the optimization target and reduce the danger of overfitting. Their use leads to higher quality, more chemically accurate models.

Most current refinement programs (Afonine et al., 2012; Murshudov et al., 2011; Sheldrick, 2015; Bricogne et al., 2011) employ a set of covalent geometry restraints first proposed by Engh and Huber in 1991 and later augmented and improved in 2001 (Engh \& Huber, 1991, 2001). This set of restraints is based on a survey of accurate high-resolution small-molecule crystal structures from the Cambridge Structural Database (Groom et al., 2016) and includes restraints on interatomic bond lengths, bond angles and $\omega$ torsion angles. In addition, parameters are added to enforce proper chirality and planarity, multiple-minimum targets for backbone and sidechain torsion angles, and repulsive terms to prevent steric overlap between atoms. Those terms are defined from smallmolecule and high-resolution macromolecular crystal structure data and from interaction-specified van der Waals radii. They are very similar, but not identical, between refinement programs.

The Engh and Huber restraints function reasonably well, while the additional terms have been gradually improved, but a number of limitations have been identified over the years. Some of these limitations include a lack of adjustability to differences in local conformation, protonation and hydrogen bonding and to their changes during refinement, incomplete or inaccurate atom types and parameters for ligands, carbohydrates and covalent modifications, the use of only repulsive and not attractive steric terms, the omission of explicit $\mathrm{H}$ atoms and their interactions, misleading targets resulting from experimental averaging artifacts, inaccurate dihedral restraints, and a lack of awareness of electrostatic and quantum dispersive interactions, with a consequent lack of accounting for hydrogen-bonding cooperativity (Priestle, 2003; Touw \& Vriend, 2010; Davis et al., 2003; Moriarty et al., 2014; Tronrud et al., 2010).

Phenix (Liebschner et al., 2019) includes a built-in system for defining ligand parameters (Moriarty et al., 2009) that by default restrains the explicit $\mathrm{H}$ atoms at electron-cloud center positions for X-ray crystallography and optionally at nuclear positions for neutron crystallography (Williams, Headd et al., 2018). The addition of the Conformation Dependent Library (CDL; Moriarty et al., 2014), which makes backbone bond lengths and angles dependent on $\varphi, \psi$ values, has improved the models obtained from refinement at all resolutions, and thus is the default in Phenix refinement (Moriarty et al., 2016). Similarly, Phenix uses ribose-pucker- and base-type-dependent torsional restraints for RNA (Jain et al., 2015). For bond lengths and angles, protein side chains continue to use standard Engh and Huber restraints, while RNA/DNA use early values (Parkinson et al., 1996) with a few modifications. This use of combined restraints is here designated $\mathrm{CDL} / \mathrm{E} \& \mathrm{H}$.

An alternative approach is the use of geometry restraints based on the all-atom force fields used for molecular-dynamics studies. This is not a novel idea. In fact, some of the earliest implementations of refinement programs employed molecularmechanics force fields (Jack \& Levitt, 1978; Brünger et al., 1987, 1989). However, at the time, restraints derived from the coordinates of ideal fragments (Tronrud et al., 1987; Hendrickson \& Konnert, 1980) were found to provide better refinement results. The insufficiency of molecular-mechanicsbased restraints was mainly attributed to two factors: an inaccurate representation of chemical space because of too few atom types, and biases in conformational sampling resulting from unshielded electrostatic interactions. Subsequently, however, the methods of molecular dynamics and the corresponding force fields have seen significant development and improvement. Current force fields contain more atom types and are easily adjustable as needed. They are typically parameterized against accurate quantum-mechanical calculations, which was not feasible just a few years ago, as well as using more representative experimental results. Significant methodological advances, such as the development of the particle mesh Ewald method (York et al., 1993; Darden et al., 1993) for the accurate calculation of crystalline electrostatics and improved temperature- and pressure-control algorithms, have greatly increased accuracy. Modern force fields have been shown to agree well with experimental data (Zagrovic et al., 2008; van Gunsteren et al., 2008; Showalter \& Brüschweiler, 2007; Grindon et al., 2004; Bowman et al., 2011), including crystal diffraction data (Cerutti et al., 2008, 2009; Janowski et al., 2013, 2015; Liu et al., 2015).

We have made it possible to use the Amber molecularmechanics force field as an alternative source of geometry restraints to those from $\mathrm{CDL} / \mathrm{E} \& \mathrm{H}$. Here, we present an integration of the Phenix software package for crystallographic refinement, phenix.refine (Afonine et al., 2012), and the Amber software package (Case et al., 2018) for molecular dynamics. We present results of paired refinements for 22544 structures and compare Amber with traditional refinement in terms of model quality, chemical accuracy and agreement with experimental data, studied both for overall statistics and for representative individual examples. We also describe the implementation and discuss future directions.

\section{Methods}

\subsection{Code preparation}

The integration of the Amber code into phenix.refine uses a thin client. Amber provides a Python API to its sander module, so that a simple 'import sander' Python command allows Phenix to obtain Amber energies and forces through a method call. At each step of coordinate refinement, Phenix expands the asymmetric unit coordinates to a full unit cell (as required by sander), combines energy gradients returned from Amber (in place of those from its internal geometric restraint routines) with gradients from the X-ray target function, and uses these forces to update the coordinates. Alternate conformers can take advantage of the 'locally enchanced sampling' (LES) facility in sander: atoms in single-conformer regions interact with multiple-copy regions via the average 
energy of interaction, while different copies of the same group do not interact among themselves (Roitberg \& Elber, 1991; Simmerling et al., 1998).

The Amber files required are created by a preliminary AmberPrep program that takes a PDB file as input. It creates both a parameter-topology (prmtop) file used by Amber and a new PDB file containing a complete set of atoms (including hydrogens and any missing atoms) needed to perform forcefield calculations. If requested, alternate conformers present in the input PDB file can be translated into sander LES format. For most situations, AmberPrep does not require the user to have any experience with Amber or with molecular mechanics; less-common situations (described in the supporting information) require some familiarity with Amber. All of the code required for both the AmberPrep and phenix.refine steps is included in the current major release, v.1.16-3549, and subsequent nightly builds of Phenix.

\subsection{Structure selection and overall refinement protocol}

To compare refinements using Amber against traditional refinements with $\mathrm{CDL} / \mathrm{E} \& \mathrm{H}$ restraints, structures were selected from the Protein Data Bank (PDB; Burley et al., 2019) using the following criteria. Entries must have untwinned experimental data available that are at least $90 \%$ complete. For each entry, $R_{\text {free }}$ was limited to a maximum of $35 \%, R_{\text {work }}$ to $30 \%$ and $R_{\Delta}\left(R_{\text {free }}-R_{\text {work }}\right)$ to a minimum of $1.5 \%$. The lowest resolution was set at $3.65 \AA$. Entries containing nucleic acids were excluded.

Coordinate and experimental data files were obtained directly from the PDB and inputs were prepared via the automated AmberPrep program (see Section 2.1). Entries containing complex ligands were included if the file-preparation program AmberPrep was able to automatically generate and include the ligand geometry data; this generally excludes ligands containing covalent connections to the protein or with metal atoms. Details of the internals of AmberPrep will be described elsewhere. Resolution bins (set at $0.1 \AA$ ) with less than ten refinement pairs were eliminated to reduce the noise

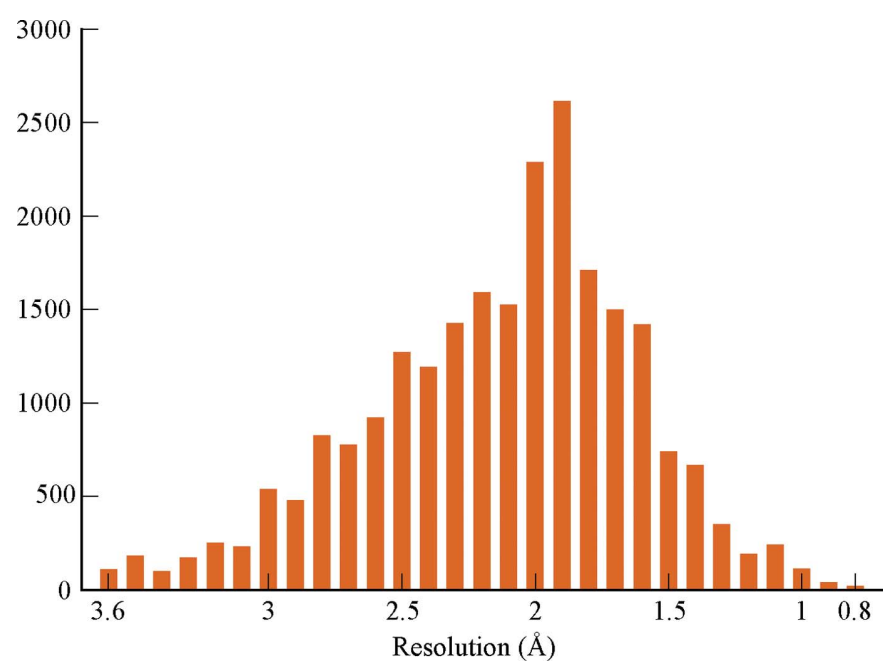

Figure 1

Distribution of refined structures across resolution bins. caused by limited statistics. Complete graphs are included in the supporting information. The resulting $22000+$ structures had experimental data resolutions between 0.8 and $3.6 \AA$, with most of the structures in the 1.2-3.0 $\AA$ range (see Fig. 1).

Each model was then subjected to ten macrocyles of refinement using the default strategy in phenix.refine for reciprocal-space coordinate refinement, with the exception that real-space refinement was turned off. By default, the first macrocycle uses a least-squares target function and the rest use maximum likelihood. Other options applied to both CDL/ $\mathrm{E} \& \mathrm{H}$ and Amber refinements included optimization of the weight between the experimental data and the geometry restraints. This protocol was performed in parallel, once using $\mathrm{CDL} / \mathrm{E} \& \mathrm{H}$ and once using Amber geometry restraints. In addition, $\mathrm{C}^{\beta}$ pseudo-torsion restraints were not included in the restraints model. Explicit parameter settings are included in the supporting information. Only one copy of each alternate conformation was considered initially (i.e. alternative location A). The final files are available by contacting the corresponding author.

The quality of the resulting models was assessed numerically using MolProbity (Williams, Headd et al., 2018) available in Phenix (Adams et al., 2010), by cpptraj (Roe \& Cheatham, 2013) available in AmberTools (Case et al., 2018) and by visual inspection with electron-density and validation markup in KiNG (Chen et al., 2009). All-atom dots for Fig. 10 were counted in Mage (Richardson \& Richardson, 2001) and Figs. 5-9 were made in KiNG. To avoid typographical ambiguity, PDB codes are given here in lower case for all letters except $\mathrm{L}$ (for example 1nLs; Moriarty, 2015).

\subsection{Weight-factor details}

The target function optimized in phenix.refine reciprocalspace atomic coordinate refinement is of the general form

$$
T_{x y z}=w * T_{\exp }+T_{x y z \_ \text {restraints }},
$$

where all of the terms are functions of the atomic coordinates, $T_{x y z}$ is the target residual to be minimized, $T_{\mathrm{exp}}$ is a residual between the observed and model structure factors and quantifies agreement with experimental data, $T_{x y z \text { restraints }}$ is the residual of agreement with the geometry restraints and $w$ is a scale factor that modulates the relative weight between the experimental and the geometry restraint terms. In traditional refinement $T_{x y z \_r e s t r a i n t s}$ is calculated using the set of CDL/ E\&H restraints,

$$
T_{x y z}=w * T_{\exp }+T_{\mathrm{CDL} / \mathrm{E} \& \mathrm{H}} .
$$

To implement Phenix-Amber we substitute this term with the potential energy calculated using the Amber force field,

$$
T_{x y z}=w * T_{\exp }+E_{\text {AmberFF }}
$$

where the Amber term is intentionally represented now by an $E$ to emphasize that we directly incorporate the full potential energy function calculated in Amber using the ff14SB force field (Maier et al., 2015). 
In a standard default Phenix refinement, the weight $w$ is a combination of a value based on the ratio of gradient norms (Brünger et al., 1989; Adams et al., 1997) and a scaling factor that defaults to 0.5 . This initial weight can be optimized using a procedure described previously (Afonine et al., 2011). This procedure uses the results of ten refinements with a selection of weights, considering the bond and angle r.m.s.d., the $R$ factors and validation statistics to determine the best weight for the specific refinement at each of the ten macrocycles. The same procedure was used to estimate an optimal weight for the Phenix-Amber refinements. (If faster fixed-weight refinements are desired, we have found that a scaling factor of 0.2 , rather than 0.5 , scales the Amber gradients to be close to those from the $\mathrm{CDL} / \mathrm{E} \& \mathrm{H}$ restraints, allowing the simpler, default, weighting scheme in phenix.refine to be used.)

\section{Results}

\subsection{Full-data-set score comparisons}

On average, the Phenix-Amber combination produced slightly higher $R_{\text {work }}$ and $R_{\text {free }}$ values (Fig. 2) but higher quality models (Fig. 3). The increase in $R$ factors is most pronounced in the 1.8-2.8 $\AA$ range. This is a result of the weight-optimization procedure having different limits for optimal weight in this resolution range. The increase was less for $R_{\text {free }}$ than $R_{\text {work }}$, such that $R_{\Delta}$ is less for refinements using Amber gradients. The uncertainty in the $R_{\text {free }}$ for $95 \%$ of refinements calculated using equation (13) of Tickle et al. (2000) is less than 0.032. At $2 \AA$ resolution, this equates to an uncertainty of $0.7 \%$, which is approximately the same as the difference in the average $R_{\text {free }}$ values of $23.0 \%$ and $23.6 \%$ for Phenix and Phenix-Amber, respectively.

The Phenix-Amber refinements exhibited improved (lower) MolProbity scores and contained fewer clashes between atoms. Plots show the mean of the values in the $0.1 \AA$ resolution bin as well as the $95 \%$ confidence level of the standard error of the mean (SEM). The MolProbity clashscores are particularly striking: for refinement using CDL/ $\mathrm{E} \& \mathrm{H}$ restraints the clashscores steadily increase as resolution worsens, often resulting in very high numbers of steric clashes. On the other hand, the mean clashscore with Amber restraints appears to be nearly independent of resolution and remains consistent at about 2.5 clashes per 1000 atoms across all resolution bins. The SEM range is non-overlapping at worse than $1 \AA$, indicating that the Amber force field is producing better geometries at mid to low resolution. There are more favored Ramachandran points (backbone $\varphi, \psi$ ) and fewer Ramachandran outliers for the Phenix-Amber refinements. This difference is most marked for resolutions worse than $2 \AA$. Phenix-Amber refinement also improves (lowers) the number of rotamer outliers but does not differentiate via the SEM, and increases the proportion of hydrogen bonds. While the rotamer outlier results remain similar, the hydrogen-bonding results have a large difference at worse than $2 \AA$, resulting in nearly double the bonds near $3 \AA$. Common to all the plots is a change near $2 \AA$, where the weight-optimization procedure common to both CDL/E\&H and Amber refinement loosens the weight on geometry restraints somewhat to allow more deviations at resolutions where the data are capable of unambiguously showing them. Bond and angle r.m.s.d. comparisons are less pertinent as the force fields do not have ideal values for parameterizations and comparing the PhenixAmber bonds and angles with the CDL/E\&H values is not a universal metric. The curious can see the plots in Supplementary Fig. S1. Overall, the improvement with Amber is substantial in the lower resolution refinements.

One validation metric that is worse for Phenix-Amber refinements is the number of outliers for $\mathrm{C}^{\beta}$ positions. Both the mean and the SEM show clear differentiation. The $\mathrm{C}^{\beta}$ deviation $(\mathrm{C} \beta \mathrm{d})$ is the distance between the modeled $\mathrm{C}^{\beta}$ and an ideal $\mathrm{C}^{\beta}$, which is a combined measure of distortion in the tetrahedron around the $\mathrm{C}^{\alpha}$ atom. The ideal position is calculated by averaging the $\mathrm{N}-\mathrm{C}-\mathrm{C}^{\alpha}-\mathrm{C}^{\beta}$ and $\mathrm{C}-\mathrm{N}-\mathrm{C}^{\alpha}-\mathrm{C}^{\beta}$ improper dihedrals and correcting the bond length, which allows for the effect of a non-ideal $\tau$ angle (Lovell et al., 2003). With traditional $\mathrm{E} \& \mathrm{H}$ restraints the $\mathrm{C} \beta \mathrm{d}$ is quite robustly sensitive to incompatibility between how the backbone and side-chain conformations have been modeled. For CDL/E\&H refinements, however, the percentage of $\mathrm{C} \beta \mathrm{d}$ outliers ( $>0.25 \AA$ ) is negligible for low and mid resolutions, only increasing to $0.2 \%$ at higher resolutions (see Fig. 4). This is in line with $\mathrm{CDL} / \mathrm{E} \& \mathrm{H}$ providing tight geometrical restraints out to $\mathrm{C}^{\beta}$ at most resolutions, but loosened somewhat at better than $2 \AA$ resolution, where there is sufficient experimental information to move an angle away from ideal. Note that explicit $\mathrm{C}^{\beta}$ restraints were turned off for all Phenix refinements and that the Amber force field does not have an explicit $\mathrm{C}^{\beta}$ term; however, if all angles around the $\mathrm{C}^{\alpha}$ atom are kept ideal then the $\mathrm{C}^{\beta}$ position will also be ideal even if it is incorrectly positioned in the structure. The following section analyses specific local examples where output structures show

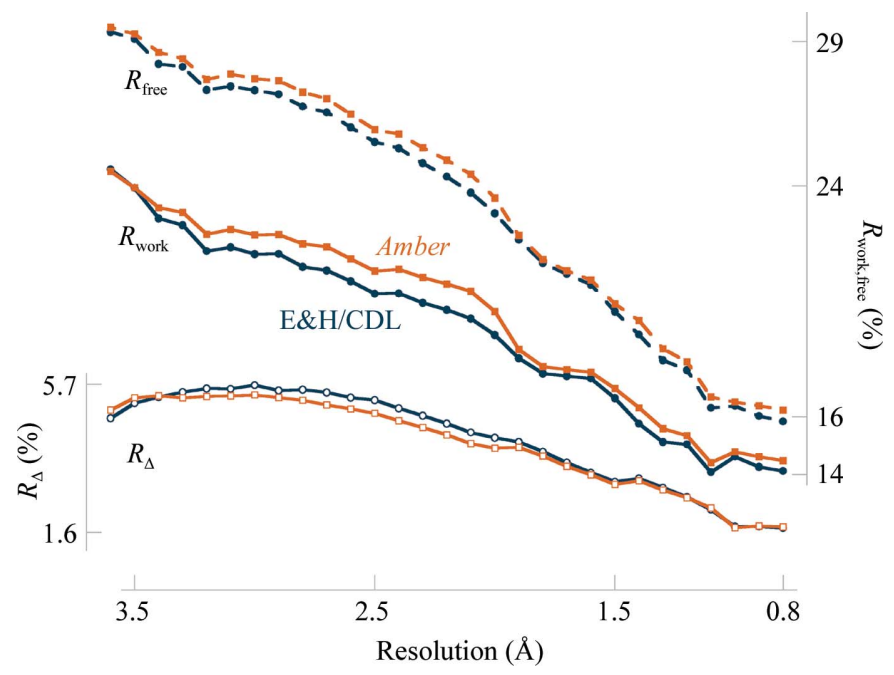

Figure 2

$R$ factors of optimized weight refinements and $R_{\text {free }}-R_{\text {work }}\left(R_{\Delta}\right)$ versus resolution (values averaged in each resolution bin). Vertical axes are in \% with the $R_{\Delta}$ axis on the left. E\&H/CDL values are plotted in dark blue and those for Amber in burnt orange. 
differences for either the positive or the negative trends seen in the overall comparisons, in order to understand their nature, causes and meaning across resolution ranges.

\subsection{Examination of individual examples}

As noted above, in comparison with the CDL/E\&H restraint refinements, the Phenix-Amber refinements have much higher percentages of $C^{\beta}$ deviation outliers, increasing at the low-resolution end to more than $1 \%$ of $\mathrm{C}^{\beta}$ atoms. Amber refinement also has more bond-length and angle outliers. The following examines a sample of cases at high, mid and lower resolutions to understand the starting-model characteristics and refinement behavior that produce these differences.

3.2.1. High resolution: waters, alternates, $C \beta d$ outliers and atoms in the wrong peak. In the high-resolution range (better than $1.7 \AA$ ), it appears that the commonest problems that are not easily correctable by refinement are caused either by modeling the wrong atom into a density peak or by incorrect modeling, labeling or truncation of alternate conformations. Such problems are usually flagged in validation either by allatom clashes, by $\mathrm{C}^{\beta}$ deviations and sometimes by bad bond lengths and angles. (For the high-resolution examples described here, we used the LES procedure outlined above to model alternative conformers in the Phenix-Amber refinements.)

Fig. 5(a) shows a case in which a water molecule had been modeled in an electron-density peak that should really be an $\mathrm{N}$ atom of an arginine guanidinium. CDL/E\&H refinement (Fig. $5 b$ ) corrected the bad geometry at the cost of moving the guanidinium even further out of density; Amber refinement changed the orientation of the guanidinium but made no overall improvement (Fig. 5c); all three versions have a bad clash. If the water were deleted then either refinement method would undoubtedly do an excellent job (Fig. $5 d$ ). This type of problem is absent at low resolution, where waters are not modeled, but occurs quite often at both high and mid resolution for other branched side chains, for Ile $\mathrm{C}^{\delta}$ (for example, Ile195 in PDB entry 3js8) and even occasionally for Trp (for example, TrpB170 in PDB entry 1qw9).

$\mathrm{C}^{\beta} \quad$ deviation outliers $(\geq 0.25 \AA$ ) are often produced by side-chain alternates with quite different $\mathrm{C}^{\beta}$ positions but with no associated alternates defined along the backbone. Since the tetrahedron around $\mathrm{C}^{\alpha}$ should be nearly ideal, this treatment almost guarantees bad geometry. The rather simple solution, implemented in Phenix, is to define alternates for all atoms until the $i+1$ and $i-1 \mathrm{C}^{\alpha}$ atoms, as in the 'backrub' motion (Davis et al., 2006). PDB entries $1 \mathrm{dy} 5$, 1 gwe and $1 \mathrm{nLs}$ each have a number of such cases. Figs. 6(a) and $6(b)$ show Ser215 in PDB entry $1 \mathrm{nLs}$, initially with an outlier $\mathrm{C} \beta \mathrm{d}$, a distance of $0.49 \AA$ between the two $\mathrm{C}^{\beta}$ atoms and a single $\mathrm{C}^{\alpha}$ atom. $\mathrm{CDL} / \mathrm{E} \& \mathrm{H}$ refinement pulls the $\mathrm{C}^{\beta}$ atoms to be only $0.23 \AA$ apart, avoiding a $\mathrm{C} \beta \mathrm{d}$ with only slightly worse fit to the density; Amber reduces the

Figure 3

Comparison plots of model-quality measures versus resolution for Amber (burnt orange) versus CDL/E\&H (dark blue) refinements with error bars depicting the $95 \%$ confidence level of the standard error of the mean. The MolProbity score is a combination of all-atom clashscore and Ramachandran favored and rotamer outliers, weighted to approximate the expected score at the resolution of the structure. The hydrogen-bond fraction is calculated using cpptraj per 1000 atoms in the model. For all six plots, Amber (burnt orange) differs in the better direction. 
$\mathrm{C} \beta \mathrm{d}$ only slightly, but it does keep this flag of an underlying problem. When alternates are defined for the backbone peptides, both systems improve.

Worse cases occur where one or both alternates have been fitted incorrectly as well as not being expanded along the backbone appropriately. Fig. 6(c) shows Thr196, with a huge $\mathrm{C} \beta \mathrm{d}$ of $0.88 \AA$ (sphere not shown) and very poor geometry because alternate $\mathrm{B}$ was fitted incorrectly (just as a shift of alternate $\mathrm{A}$ rather than as a new rotamer). This time even $\mathrm{CDL} / \mathrm{E} \& \mathrm{H}$ refinement produces a $\mathrm{C} \beta \mathrm{d}$ outlier, but smaller

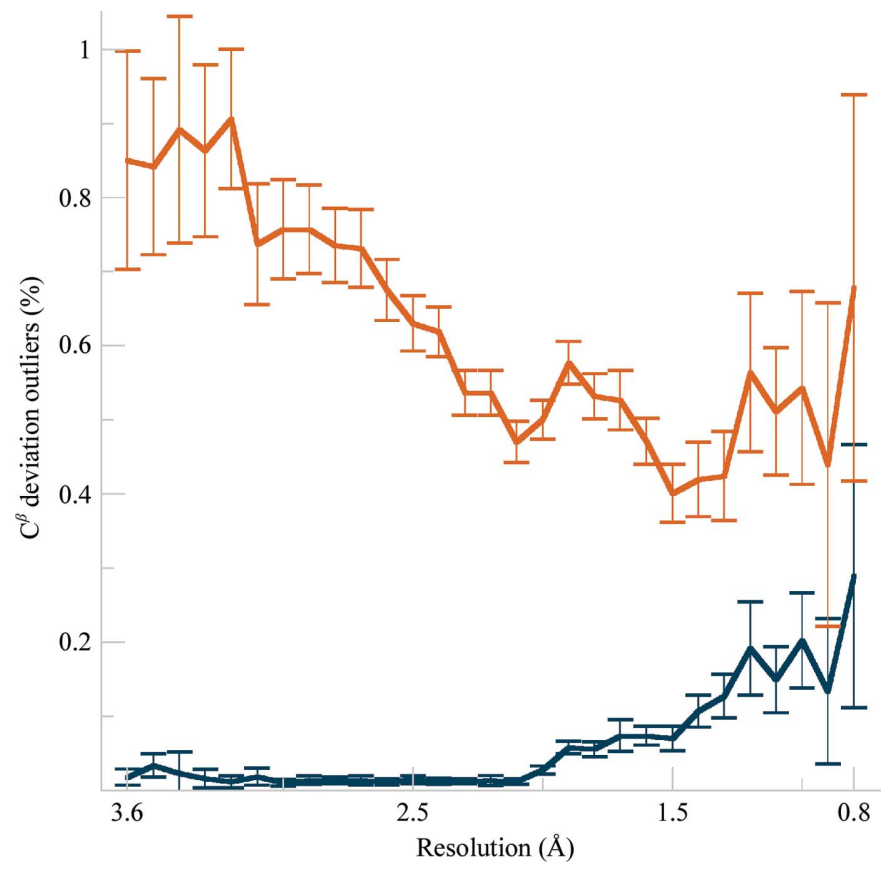

Figure 4

Fraction of $\mathrm{C}^{\beta}$ deviations (in \%) per $\mathrm{C}^{\beta}$ atom as a function of resolution for the CDL/E\&H (dark blue) and Amber (burnt orange) refinements. Values are averaged in each bin of resolution, with the error bars showing the $95 \%$ confidence level of the standard error of the mean. than that for Amber. Fig. 6(d) shows the excellent Amber result after the misfitting of alternate $\mathrm{B}$ was approximately corrected.

3.2.2. Mid resolution: backward side chains and rare conformations. An even commoner case at both high and mid resolutions where the wrong atom is fitted into a density peak is a backward-fitted $\mathrm{C}^{\beta}$-branched residue, which is well illustrated by a very clear Thr example in PDB entry $1 \mathrm{bkr}$ at $1.1 \AA$ resolution (Fig. 7a). Thr101 is a rotamer outlier (gold) on a regular $\alpha$-helix with a C $\beta$ d of $0.63 \AA$. The deposited Thr101 also has a bond-angle deviation of $13.5 \sigma$, clashes at the $\mathrm{C}^{\gamma}$ methyl, its $\mathrm{C}^{\beta}$ is out of density, $\mathrm{O}^{\gamma}$ is in the lower peak and $\mathrm{C}^{\gamma}$ is in the higher peak. It is shown in Fig. 7 with $1.6 \sigma$ and $4 \sigma$ $2 m F_{\mathrm{o}}-D F_{\mathrm{c}}$ contours (but without $\mathrm{C}^{\beta}$ deviation and angle markups for clarity). This mistake was not obvious because anisotropic $B$ factors were used too early in the modeling, resulting in the Thr $\mathrm{C}^{\beta}$ being refined to a 6:1 aniso-axis ratio that covered both the modeled atom and the real position. The figures show the density as calculated with isotropic $B$ factors.

Given this difficult problem for automated refinement, each of the two target functions reacts very differently. Both refinements still have the $\mathrm{C}^{\gamma}$ methyl clashing with a helix backbone $\mathrm{CO}$ in good density, which is very diagnostic of a problem with the $\mathrm{C}^{\gamma}$ atom. It is indeed the wrong atom to have in this peak, as is also shown by the relative peak heights. The CDL/E\&H refinement (Fig. $7 b$ ) achieves tight geometry and a good rotamer, moving the $\mathrm{C}^{\beta}$ atom into its correct density peak, but pays the price for not correcting the underlying problem by swinging the $\mathrm{O}^{\gamma}$ atom out of density. The Amber refinement (Fig. 7c) achieves an atom in each of the three sidechain density peaks, but pays the price for not correcting the underlying problem by having the wrong chirality at the $\mathrm{C}^{\beta}$ atom. It still also has bond-angle outliers, which may be a sign of unconverged refinement.

The original PDB entry, the CDL/E\&H refinement and the Amber refinement structures for Thr101 are all very badly

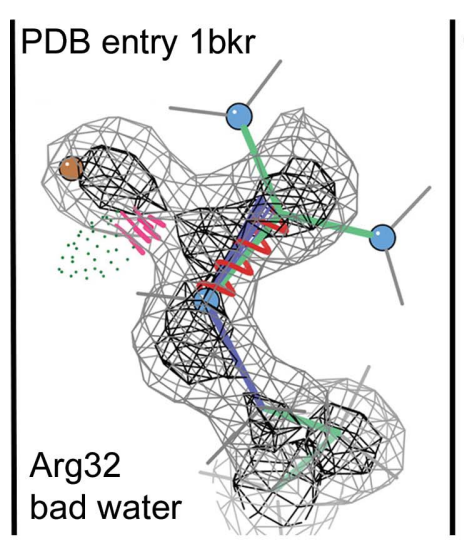

(a)

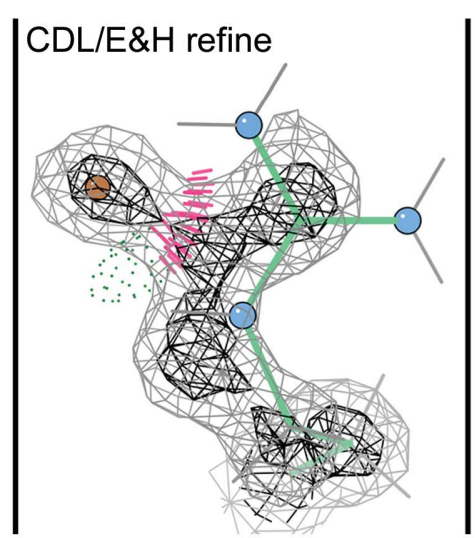

(b)

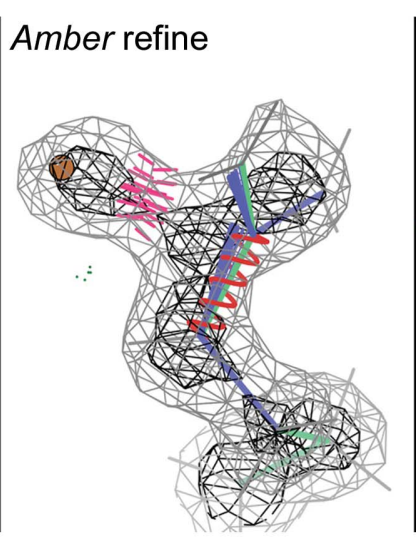

(c)

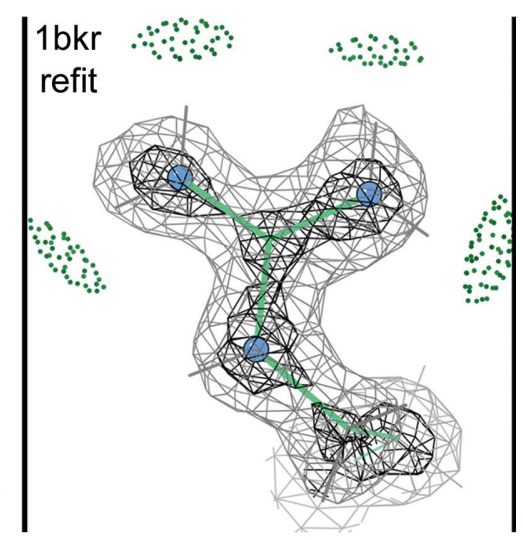

$(d)$

Figure 5

Differing responses of $\mathrm{CDL} / \mathrm{E} \& \mathrm{H}$ versus Amber refinement to the misfitting of a water into what should be a side-chain $\mathrm{N}$ atom in an arginine residue. Neither result here is acceptable, but if the incorrect water is deleted $(d)$ then both methods do a very good job of moving the guanidinium correctly back into its density. MolProbity markup for Figs. 5-10: clusters of hot pink spikes represent clashes, pillows of green dots represent hydrogen bonds, red or blue springs or fans represent larger or smaller bond-length or angle outliers, magenta spheres represent $\mathrm{C}^{\beta}$ deviations, gold side chains represent rotamer outliers, green $\mathrm{C}^{\alpha}-\mathrm{C}^{\alpha}$ lines represent Ramachandran outliers and magenta lines along the CO-CO dihedral represent $C a B L A M$ outliers. Relevantly moving $\mathrm{O}$ or $\mathrm{N}$ atoms are emphasized with red or blue spheres. 
wrong, but each in an entirely different way. The deposited model, PDB entry $1 \mathrm{bkr}$, looks very poor by traditional model validation, but has a misleadingly good density correlation given the extremely anisotropic $\mathrm{C}^{\beta} B$ factor. The CDL/E\&H output looks extremely good on traditional validation except for the clashes and would show a lowered but still reasonable density correlation; however, it is the most obviously wrong upon manual inspection. The Amber output has clashes and currently has modest bond-angle outliers, but it fits the density very closely, making it difficult to identify as incorrect by visual inspection. The problem could be recognized automatically by a simple chirality check. As shown in Fig. 7(d), Thr101 was rebuilt quickly in KiNG with the p rotamer and a small backrub motion. Either Phenix-CDL/E\&H or PhenixAmber refinement would do a very good job from such a rough refit with the correct atoms near the right places.

At mid resolution, there are also other rotamers and backbone conformations fitted into the wrong local minimum, and thus difficult to correct by minimization refinement methods, but not always flagged by $\mathrm{C}^{\beta}$ deviations or other outliers. Some of these, such as cis-nonproline peptides (Williams, Videau et al., 2018) or very rare rotamers (Hintze et al., 2016), can be avoided by considering their highly unfavorable prior probabilities. Others would require explicit sampling of the multiple minima.

3.2.3. Lower resolution: peptide orientations with CaBLAM and C $\beta$ d outliers. At low resolution $(2.5-4 \AA)$, no waters or alternates are modeled. All other problems continue, but an additional set of common local misfittings occur because the broad electron density is compatible with significantly different models. PDB entry 1 xgo at $3.5 \AA$ resolution is an excellent case for testing in this range, because it was solved independently from the $1.75 \AA$ resolution structure with PDB code 1xgs: the same molecule in a different space group. CDL/E\&H refinement shows no $\mathrm{C} \beta \mathrm{d}$ outliers, but Amber refinement shows six. Comparison with PDB entry $1 \mathrm{xgs}$ shows that each of the $\mathrm{C} \beta \mathrm{d}$ residues has the side chain, the backbone or both in an incorrect local-minimum

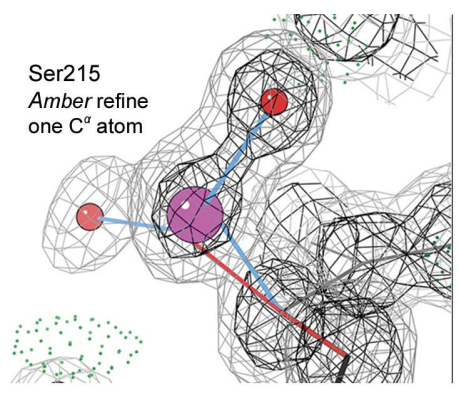

(a)

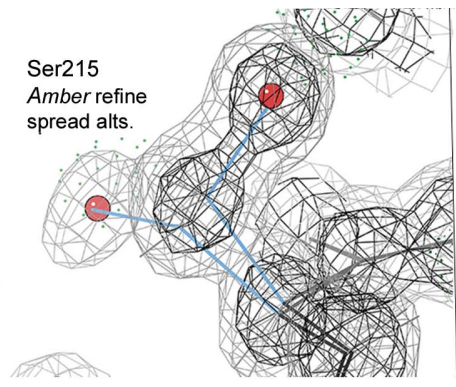

(b)

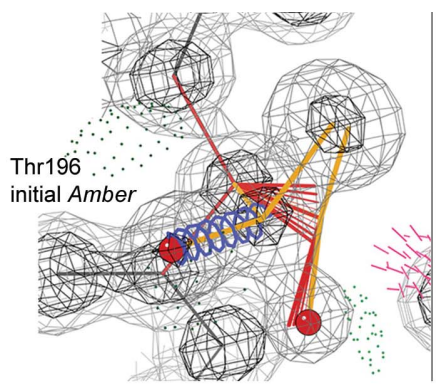

(c)

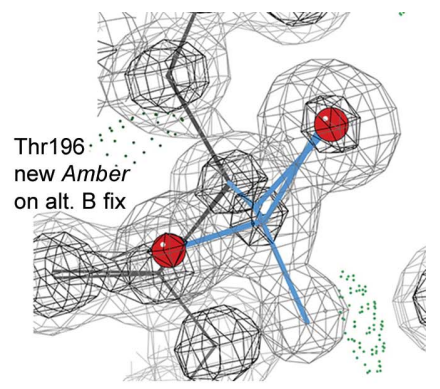

(d)

Figure 6

At high resolution, $\mathrm{C}^{\beta}$ deviation outliers are most often due to problems with alternate conformations. (a) Amber refinement using the original Ser215 alternates in PDB entry $1 \mathrm{nLs}$, which have widely separated positions for $\mathrm{C}^{\beta}$ but only a single $\mathrm{C}^{\alpha}$ atom. $(b)$ Amber refinement after the definition of alternates has been spread to include the $\mathrm{C}^{\alpha}$ and both adjoining peptides. (c) Amber refinement of the original Thr196 of PDB entry $1 \mathrm{nLs}$, where alternate B had been fitted backwards; there is bad covalent geometry and a huge C $\beta \mathrm{d}$ of $0.88 \AA$ (sphere not shown). (d) The good Amber result after alternate $\mathrm{B}$ was refitted in the correct rotamer so that all atoms match the density.

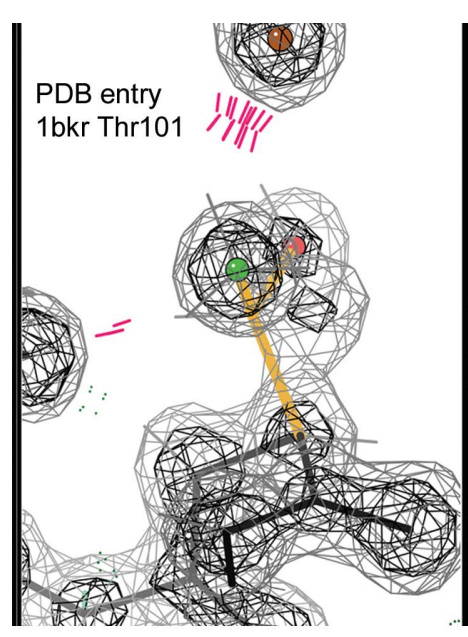

(a)

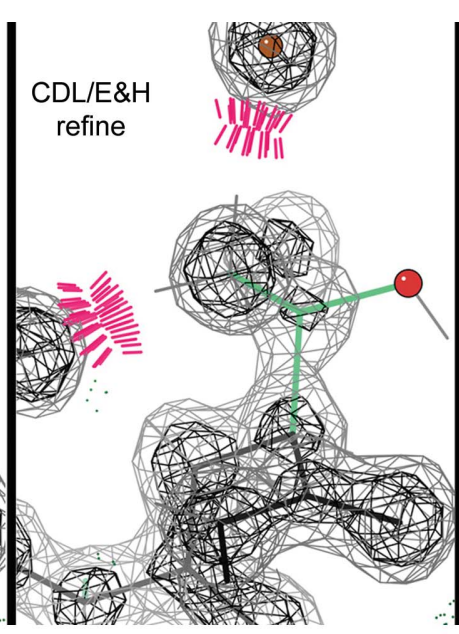

(b)

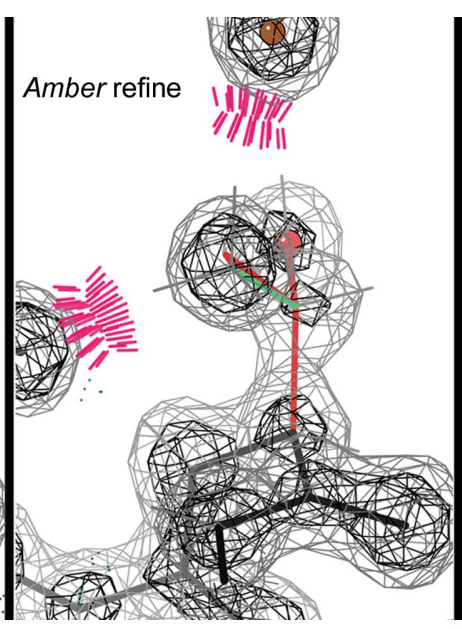

(c)

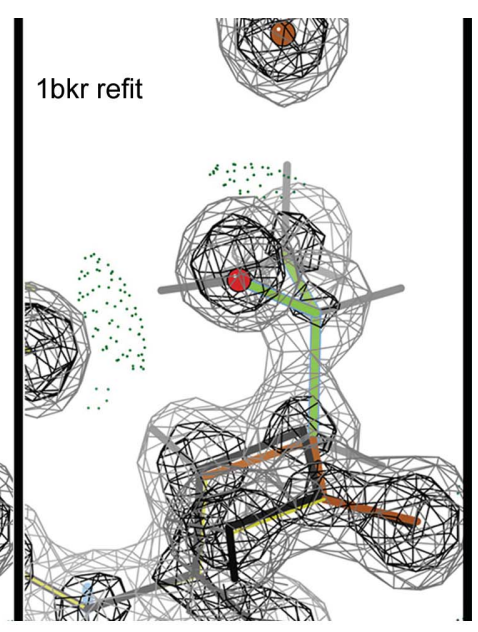

(d)

Figure 7

Unacceptable ways of getting rid of a $\mathrm{C}^{\beta}$ deviation without fixing the actual problem. (a) Thr101 in PDB entry $1 \mathrm{bkr}$ as deposited, with a huge C $\beta$ d of $0.63 \AA$ (not shown as a sphere because it obscures the side chain), clashes, a rotamer outlier, the heavier $\mathrm{O}^{\gamma}$ branch in the lower electron-density peak and the $\mathrm{C}^{\beta}$ out of density, all of which are caused by modeling the side chain $\chi_{1} 180^{\circ}$ backwards. $(b) \mathrm{CDL} / \mathrm{E} \& \mathrm{H}$ makes the geometry perfect but places the $\mathrm{O}^{\gamma}$ far out of density. (c) Amber places all three side-chain atoms into peaks by making the chirality at $\mathrm{C}^{\beta}$ incorrect. $(d) \mathrm{A}$ refit in the correct rotamer replaces clashes with hydrogen bonds, has no outliers and puts each atom into its correct density peak. 
conformation uncorrectable by minimization refinement methods (Richardson \& Richardson, 2018). For example, Fig. 8 shows Leu253 on a helix, with a $\mathrm{C} \beta$ d from Amber (Fig. 8c) and the different, correct PDB entry $1 \mathrm{xgs}$ Leu rotamer (Fig. $8 d)$. These $\mathrm{C} \beta \mathrm{d}$ outliers are thus a feature, not a bug, in Amber: they serve their designed validation function of flagging genuine fitting problems. However, the lack of $\mathrm{C} \beta \mathrm{d}$ outliers in the $\mathrm{CDL} / \mathrm{E} \& \mathrm{H}$ refinement is also not a defect because the tight $\mathrm{CDL} / \mathrm{E} \& \mathrm{H}$ geometry is on average quite useful at low resolution.

The $1 \mathrm{xgo}$ versus $1 \mathrm{xgs}$ comparison also illustrates many of the ways in which Amber refinement is superior at low resolution. In Fig. 8, Amber corrects a Ramachandran outlier in the helix and shows a helix backbone shape much closer to the ideal geometry of PDB entry $1 \mathrm{xgs}$ than either the deposited or the $\mathrm{CDL} / \mathrm{E} \& \mathrm{H}$ versions.
Since the backbone $\mathrm{CO}$ direction cannot be seen at low resolution, the commonest local misfitting is a misoriented peptide (Richardson et al., 2018). These can be flagged by the new MolProbity validation called CaBLAM, which tests whether adjacent $\mathrm{CO}$ directions are compatible with the local $\mathrm{C}^{\alpha}$ backbone conformation (Williams, Headd et al., 2018). Ten such cases were identified in PDB entry 1xgo for isolated single or double $C a B L A M$ outliers surrounded by correct structure as judged in PDB entry 1xgs. In six of those ten cases neither CDL/E\&H nor Amber refinement corrected the problem (His62, Thr70, Gly163, Gly193, Ala217 and Glu286; see Supplementary Fig. S2). In two cases CDL/E\&H had fewer other outliers than Amber refinement, but did not actually reorient the CO (Gly193 and the Gly163 case shown in Supplementary Fig. S3). In three of the ten cases Amber performed a complete fix, while CDL/E\&H did not provide

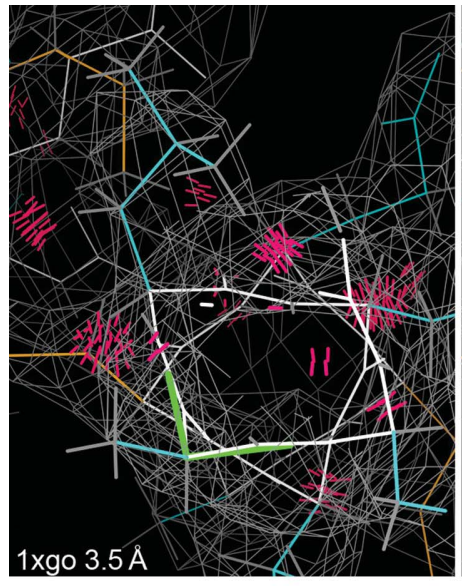

(a)

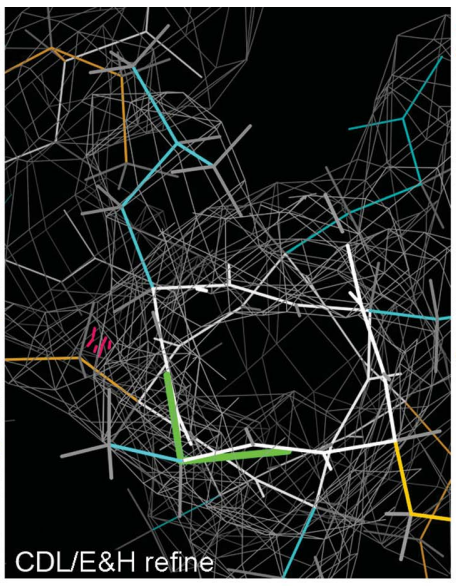

(b)

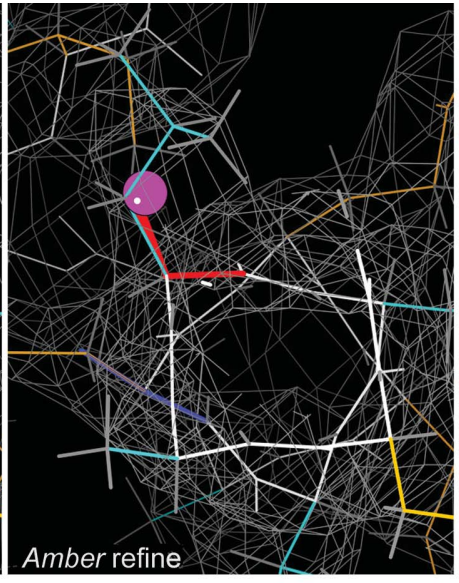

(c)

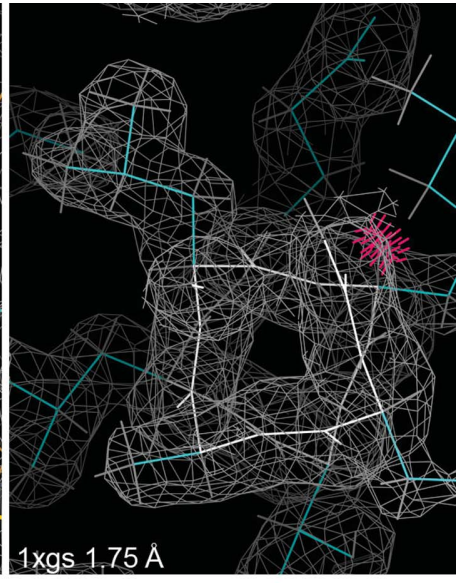

$(d)$

Figure 8

$\mathrm{A} \mathrm{C}^{\beta}$ deviation in the Amber results at $3.5 \AA$ resolution, but not for either the original or the CDL/E\&H results. (a) Leu253 in PDB entry $1 \mathrm{xgo}$ on a quite distorted helix, with many clashes and a Ramachandran outlier; the Leu rotamer is incorrect, as shown by the structure with PDB code 1xgs at $1.75 \AA$ resolution. (b) CDL/E\&H refinement fixes the clashes, but not the rotamer or Ramachandran outliers or the helix distortion. (c) Amber refinement fixes the clashes and the Ramachandran outlier, flags the incorrect Leu rotamer with a $\mathrm{C} \beta \mathrm{d}$ outlier and moves the helix conformation closer to ideal. $(d)$ Leu253 in PDB entry 1xgs at $1.75 \AA$ resolution, with a clearly correct rotamer on an ideal helix and no outliers besides one clash.

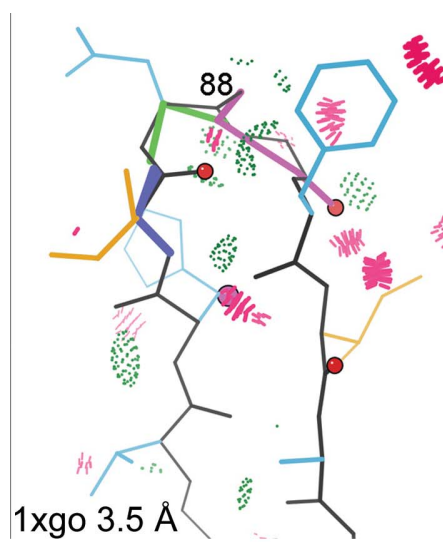

(a)

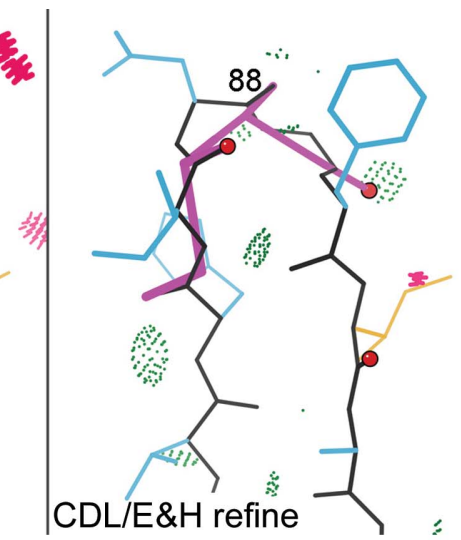

(b)

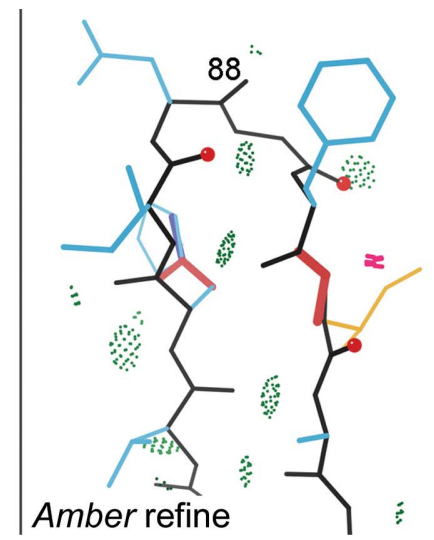

(c)

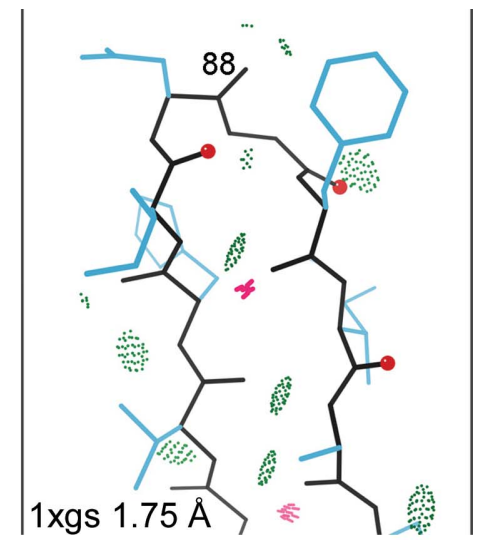

(d)

Figure 9

Two misoriented peptides in PDB entry 1xgo, flagged by Ramachandran and CaBLAM outliers (magenta outlines on the CO virtual dihedrals). (a) Residues 86-91 in the deposited structure with PDB code 1xgo. (b) CDL/E\&H result, with unchanged conformation and outliers. (c) Amber result, with several peptide orientations changed by modest amounts (red spheres on $\mathrm{CO}$ ), removing the backbone outliers and very closely matching the conformation for PDB entry 1 xgs shown in $(d)$. 


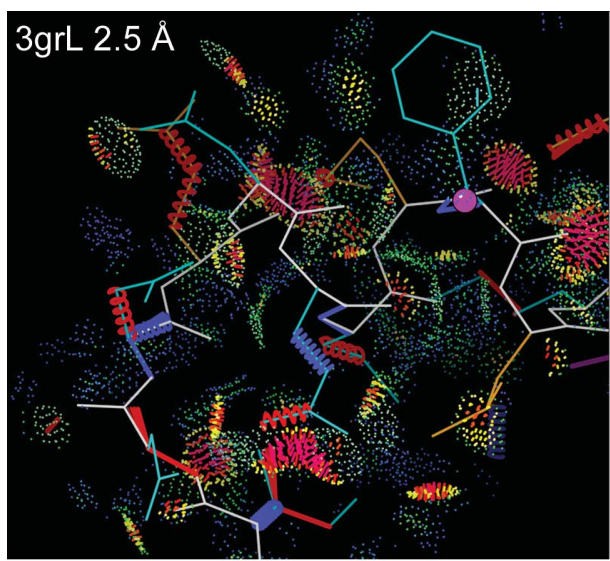

(a)

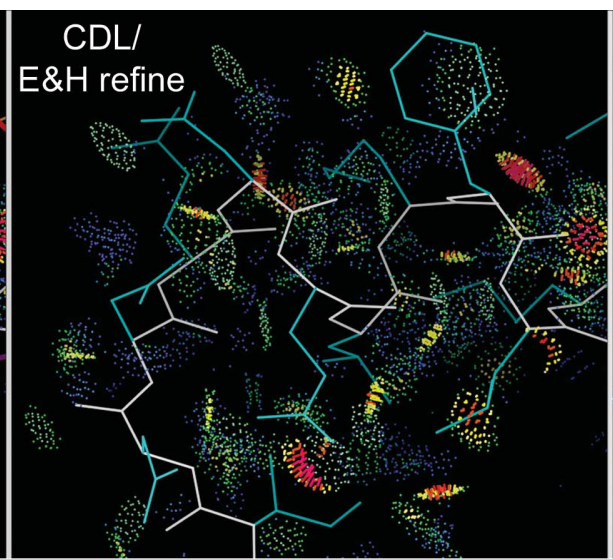

(b)

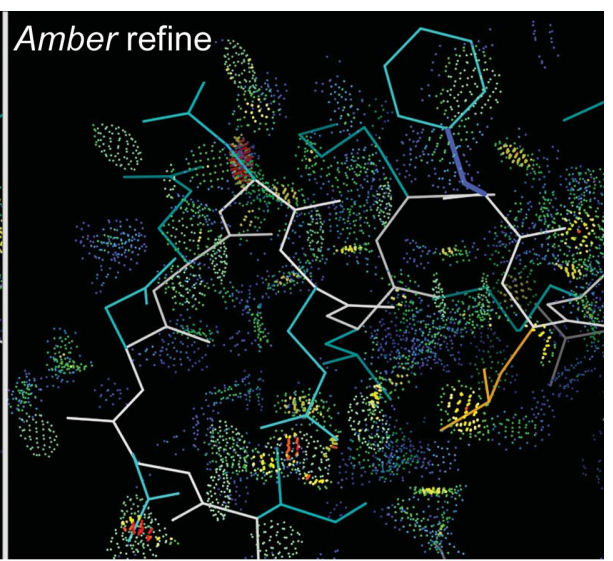

(c)

Figure 10

Amber refinement produces better hydrogen bonds and van der Waals contacts as well as removing somewhat more steric clashes. $(a)$ The Asn182 helixcap region in PDB entry $3 \mathrm{~g} 8 \mathrm{~L}$ at $2.5 \AA$ resolution, with numerous clashes and other outliers. (b) CDL/E\&H refinement makes large improvements, removing most clashes and all other outliers. (c) Amber refinement does even better, removing all clashes and most small overlaps (yellow) and optimizing to produce more hydrogen bonds and favorable van der Waals contacts (green and blue dots).

any improvement (Asp88, Gly125 and Pro266). For example, in Fig. 9, residues 86-91 of PDB entry 1xgo (Fig. 9a) have a CaBLAM outlier (magenta lines) uncorrected by CDL/E\&H refinement (Fig. 9b). However, Amber refinement (Fig. 9c) manages to shift several $\mathrm{CO}$ orientations by modest amounts (red spheres), which is sufficient to fix the $C a B L A M$ outliers and match the better backbone conformation of PDB entry 1xgs extremely closely (Fig. 9d). The Gly125 example is shown

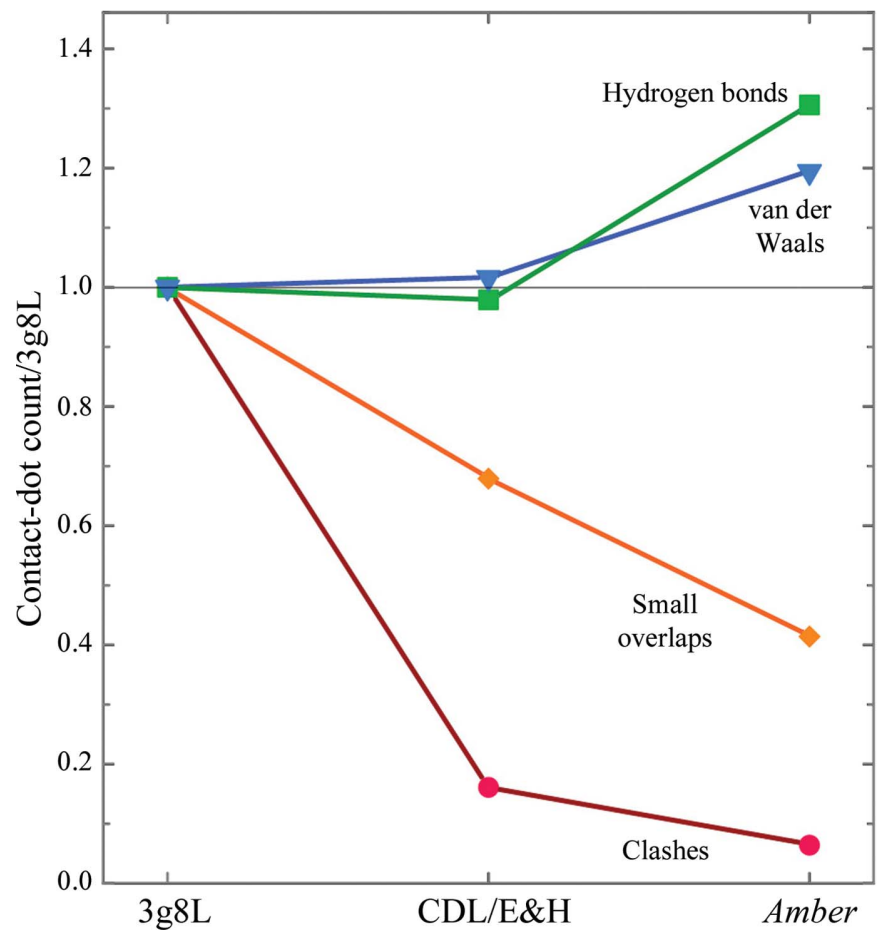

Figure 11

CDL/E\&H versus Amber improvements in steric contacts for the helixcap in PDB entry 3g8L, quantified by all-atom contact dot or spike counts measured in Mage (Richardson \& Richardson, 2001), normalized relative to the counts in the deposited structure with PDB code 3g8L. Amber changes farthest, in the right direction, for all four contact types. in Supplementary Fig. S4. Finally, in one especially interesting case (Lys22) Amber turned the $\mathrm{CO}$ about halfway up to where it should be, while CDL/E\&H made no improvement. The Amber model still has geometry outliers and further runs moved the $\mathrm{CO}$ most of the way up and removed those outliers, showing that Amber refinement had not yet fully converged in ten macrocycles (see the supporting information and Supplementary Fig. S5).

Amber refinement is especially good at optimizing hydrogen-aware all-atom sterics, as calculated by Probe (Word, Lovell, LaBean et al., 1999) with $\mathrm{H}$ atoms added and optimized by Reduce (Word, Lovell, Richardson et al., 1999). This is illustrated in Fig. 10 for PDB entry $3 \mathrm{~g} 8 \mathrm{~L}$ at $2.5 \AA$ resolution. The deposited structure of the Asn182 helix N-cap region, which has many outliers of all kinds (Fig. 10a), is improved a great deal by CDL/E\&H refinement (Fig. 10b). However, the Amber refinement (Fig. 10c) is noticeably better, with more hydrogen bonds and better van der Waals contacts, as well as fewer clashes. These improvements are plotted quantitatively in Fig. 11, as measured by a decrease in unfavorable clash spikes (red) and small overlaps (orange), with an increase in favorable hydrogen bonds (green) and van der Waals contacts (blue).

\section{Discussion}

The idea of including molecular-mechanics force fields into crystallographic refinements is not a new one, with precedents dating back to early work by Jack \& Levitt (1978) and the $X$-PLOR program (Brünger \& Karplus, 1991) developed in the 1980s. The notion that a force field could (at least in principle) encode 'prior knowledge' about protein structure continues to have a strong appeal, and efforts to replace conventional 'geometric restraints', which are very local and uncorrelated, with a more global assessment of structural quality have been explored repeatedly (see, for example, Moulinier et al., 2003; Schnieders et al., 2009). Distinguishing 
features of the current implementation include the automatic preparation of force fields for many types of biomolecules, ligands and solvent components as well as close integration with Phenix, a mature and widely used platform for refinement. This has enabled parallel refinements on more than 22000 protein entries in the PDB and allows crystallographers to test these ideas on their own systems by simply adding flags to an existing phenix.refine command line or adding the same information via the Phenix GUI. Indeed, we expect most users to 'turn on' Amber restraints after having carried out a more conventional refinement to judge for themselves the significance and correctness of the structural differences that arise. As noted in Section 3.2, an Amber refinement will often flag residues that need manual refitting in ways complementary to the cues provided by more conventional refinement.

The results presented here show that structures with improved local quality (as monitored by MolProbity criteria and hydrogen-bond analysis) can be obtained by simple energy minimization, with minimal degradation in the agreement with experimental structure factors and with no changes to a current-generation protein force field. Nevertheless, one should keep in mind that the Amber-refined structures obtained here are not very different from those found using more conventional refinement. Both methods require that most local misfittings be corrected in advance. The hope is that either sampling of explicit alternatives or else optimization using more aggressive conformational search, such as with simulated annealing or torsion-angle dynamics, may find the correct low-energy structures with good agreement with experimental data.

It is likely that further exploration of relative weights between 'X-ray' and 'energy' terms (beyond the existing and heuristic weight-optimization procedure employed here), and even within the energy terms, will become important. In principle, maximizing the joint probability arising from 'prior knowledge' [using a Boltzmann distribution, $\exp \left(-E_{\mathrm{AmberFF}} / k_{\mathrm{B}} T\right)$, for some effective temperature] and a maximum-likelihood target function (based on a given model and the observed data) is an attractive approach that effectively establishes an appropriate relative weighting. More study will be needed to see how well this works in practice, especially in light of the inevitable limitations of current force fields.

The integration of the Amber force field into the Phenix software for crystallography also paves the way for the development of more sophisticated applications. The force field can accommodate alternate conformers by using the locally enhanced sampling (LES) approach (Roitberg \& Elber, 1991; Simmerling et al., 1998); a few examples are discussed here, whilst details will be presented elsewhere. Ensemble refinement (Burnley et al., 2012) could now be performed using a full molecular-dynamics force field, thus avoiding poor-quality individual models in the ensemble. Similarly, simulated annealing could now be performed with an improved physics-based potential. Extension of the ideas presented to real-space refinement within Phenix is under way, opening a path to new applications to cryo-EM and low- resolution X-ray structures. These developments would all contribute significantly to the future of macromolecular crystallography, reinforcing the transition from a single static structure-dominated view of crystals to one in which dynamics and structural ensembles play a central important role in describing molecular function (Furnham et al., 2006; van den Bedem \& Fraser, 2015; Wall et al., 2014).

\section{Conclusions}

We have presented refinement results obtained by integrating Phenix with the Amber software package for molecular dynamics. Our refinements of over 22000 crystal structures show that refinement using the Amber all-atom molecularmechanics force field outperforms $\mathrm{CDL} / \mathrm{E} \& \mathrm{H}$ restraint refinement in many respects. An overwhelming majority of Amber-refined models display notably improved model quality. The improvement is seen across most indicators of model quality, including clashes between atoms, side-chain rotamers and peptide-backbone torsion angles. In particular, Phenix-Amber consistently outperforms standard Phenix refinement in clashscore, number of hydrogen bonds per 1000 atoms and MolProbity score. It also consistently outperforms standard refinement for Ramachandran and rotamer statistics at low resolutions and obtains approximately equal results at high (better than $2.0 \AA$ ) resolutions. Amber does run somewhat more slowly (generally taking $20-40 \%$ longer) and may take more cycles for a particular local conformation to converge completely if it is making a large local change (see the caption to Supplementary Fig. S5). It should be noted that standard refinement consistently outperforms Phenix-Amber in eliminating $\mathrm{C}^{\beta}$ deviation and other covalent-geometry outliers across all resolutions, but in most cases the Amber outliers serve to flag a real problem in the model.

As the quality of experimental data decreases with resolution, the improvement in model quality obtained by using Amber, as opposed to CDL/E\&H restraints, increases. This improvement is especially striking in the case of clashscores, which appear to be nearly independent of experimental data resolution for Amber refinements. Additional improvement is seen in the modeling of electrostatic interactions, hydrogen bonds and van der Waals contacts, which are currently ignored by conventional restraints. Improving lower resolution structures is very important, since they include a large fraction of the most exciting and biologically important current structures such as the protein/nucleic acid complexes of large, dynamic molecular machines.

No minimization refinement method, including $\mathrm{CDL} / \mathrm{E} \& \mathrm{H}$ and Amber, can in general correct local misfittings that were modeled in an incorrect local minimum conformation, especially at relatively high resolutions. At lower resolutions, where the barriers are softer, Amber can sometimes manage such a change, while $\mathrm{CDL} / \mathrm{E} \& \mathrm{H}$ still does not. It is, therefore, important and highly recommended that validation flags be consulted for the initial model and as many of the worst cases be fixed as feasible before starting the cycles of automated refinement with either target. 


\section{Software distribution}

Amber was implemented in phenix.refine and is available in v.1.16-3549 of Phenix and later. Instructions for using the phenix.refine Amber implementation are available in the version-specific documentation available with the distribution. The Amber codes are included in the Phenix distribution under the terms of the GNU lesser general public license (LGPL).

\section{Related literature}

The following references are cited in the supporting information for this article: Jorgensen et al. (1983), Joung \& Cheatham (2009), Tahirov et al. (1998) and Wang et al. (2004, 2006).

\section{Acknowledgements}

JSR thanks David Richardson for help with some aspects of the individual example analyses. The content is solely the responsibility of the authors and does not necessarily represent the official views of the National Institutes of Health, NIGMS or DOE.

\section{Funding information}

The following funding is acknowledged: National Institutes of Health (grant No. GM122086 to David A. Case; grant No. P01GM063210 to Paul D. Adams, Jane S. Richardson); Department of Energy (grant No. DE-AC02-05CH11231 to Lawrence Berkeley National Laboratory); the Phenix Industrial Consortium.

\section{References}

Adams, P. D., Afonine, P. V., Bunkóczi, G., Chen, V. B., Davis, I. W., Echols, N., Headd, J. J., Hung, L.-W., Kapral, G. J., GrosseKunstleve, R. W., McCoy, A. J., Moriarty, N. W., Oeffner, R., Read, R. J., Richardson, D. C., Richardson, J. S., Terwilliger, T. C. \& Zwart, P. H. (2010). Acta Cryst. D66, 213-221.

Adams, P. D., Pannu, N. S., Read, R. J. \& Brünger, A. T. (1997). Proc. Natl Acad. Sci. USA, 94, 5018-5023.

Afonine, P. V., Echols, N., Grosse-Kunstleve, R. W., Moriarty, N. W. \& Adams, P. D. (2011). Comput. Crystallogr. Newsl. 2, 99-103.

Afonine, P. V., Grosse-Kunstleve, R. W., Echols, N., Headd, J. J., Moriarty, N. W., Mustyakimov, M., Terwilliger, T. C., Urzhumtsev, A., Zwart, P. H. \& Adams, P. D. (2012). Acta Cryst. D68, 352-367. Agarwal, R. C. (1978). Acta Cryst. A34, 791-809.

Bedem, H. van den \& Fraser, J. S. (2015). Nat. Methods, 12, 307-318.

Berman, H. M., Westbrook, J., Feng, Z., Gilliland, G., Bhat, T. N., Weissig, H., Shindyalov, I. N. \& Bourne, P. E. (2000). Nucleic Acids Res. 28, 235-242.

Bowman, G. R., Voelz, V. A. \& Pande, V. S. (2011). J. Am. Chem. Soc. 133, 664-667.

Bricogne, G., Blanc, E., Brandl, M., Flensburg, C., Keller, P., Paciorek, W., Roversi, P., Sharff, A., Smart, O. S., Vonrhein, C. \& Womack, T. O. (2011). BUSTER. Global Phasing, Cambridge, UK.

Brünger, A. T. \& Karplus, M. (1991). Acc. Chem. Res. 24, 54-61.

Brünger, A. T., Karplus, M. \& Petsko, G. A. (1989). Acta Cryst. A45, 50-61.

Brünger, A. T., Kuriyan, J. \& Karplus, M. (1987). Science, 235, 458460.
Burley, S. K., Berman, H. M., Bhikadiya, C., Bi, C., Chen, L., Costanzo, L. D., Christie, C., Duarte, J. M., Dutta, S., Feng, Z., Ghosh, S., Goodsell, D. S., Green, R. K., Guranovic, V., Guzenko, D., Hudson, B. P., Liang, Y., Lowe, R., Peisach, E., Periskova, I., Randle, C., Rose, A., Sekharan, M., Shao, C., Tao, Y.-P., Valasatava, Y., Voigt, M., Westbrook, J., Young, J., Zardecki, C., Zhuravleva, M., Kurisu, G., Nakamura, H., Kengaku, Y., Cho, H., Sato, J., Kim, J. Y., Ikegawa, Y., Nakagawa, A., Yamashita, R., Kudou, T., Bekker, G.-J., Suzuki, H., Iwata, T., Yokochi, M., Kobayashi, N., Fujiwara, T., Velankar, S., Kleywegt, G. J., Anyango, S., Armstrong, D. R., Berrisford, J. M., Conroy, M. J., Dana, J. M., Deshpande, M., Gane, P., Gáborová, R., Gupta, D., Gutmanas, A., Koča, J., Mak, L., Mir, S., Mukhopadhyay, A., Nadzirin, N., Nair, S., Patwardhan, A., Paysan-Lafosse, T., Pravda, L., Salih, O., Sehnal, D., Varadi, M., Vařeková, R., Markley, J. L., Hoch, J. C., Romero, P. R., Baskaran, K., Maziuk, D., Ulrich, E. L., Wedell, J. R., Yao, H., Livny, M. \& Ioannidis, Y. E. (2019). Nucleic Acids Res. 47, D520-D528.

Burnley, B. T., Afonine, P. V., Adams, P. D. \& Gros, P. (2012). eLife, 1, e00311.

Case, D. A., Ben-Shalom, I. Y., Brozell, S. R., Cerutti, D. S., Cheatham, T. E. III, Cruzeiro, V. W. D., Darden, T. A., Duke, R. E., Ghoreishi, D., Gilson, M. K., Gohlke, H., Goetz, A. W., Greene, D., Harris, R., Homeyer, N., Izadi, S., Kovalenko, A., Kurtzman, T., Lee, T. S., LeGrand, S., Li, P., Lin, C., Liu, J., Luchko, T., Luo, R., Mermelstein, D. J., Merz, K. M., Miao, Y., Monard, G., Nguyen, C., Nguyen, H., Omelyan, I., Onufriev, A., Pan, F., Qi, R., Roe, D. R., Roitberg, A., Sagui, C., Schott-Verdugo, S., Shen, J., Simmerling, C. L., Smith, J., Salomon-Ferrer, R., Swails, J., Walker, R. C., Wang, J., Wei, H., Wolf, R. M., Wu, X., Xiao, L., York, D. M. \& Kollman, P. A. (2018). Amber18. University of California, San Francisco.

Cerutti, D. S., Le Trong, I., Stenkamp, R. E. \& Lybrand, T. P. (2008). Biochemistry, 47, 12065-12077.

Cerutti, D. S., Le Trong, I., Stenkamp, R. E. \& Lybrand, T. P. (2009). J. Phys. Chem. B, 113, 6971-6985.

Chen, V. B., Davis, I. W. \& Richardson, D. C. (2009). Protein Sci. 18, 2403-2409.

Darden, T., York, D. M. \& Pedersen, L. (1993). J. Chem. Phys. 98, 10089-10092.

Davis, A. M., Teague, S. J. \& Kleywegt, G. J. (2003). Angew. Chem. Int. Ed. 42, 2718-2736.

Davis, I. W., Arendall, W. B. III, Richardson, D. C. \& Richardson, J. S. (2006). Structure, 14, 265-274.

Engh, R. A. \& Huber, R. (1991). Acta Cryst. A47, 392-400.

Engh, R. A. \& Huber, R. (2001). International Tables for Crystallography, Vol. F, edited by M. G. Rossmann \& E. Arnold, pp. 382392. Dordrecht: Kluwer Academic Press.

Furnham, N., Blundell, T. L., DePristo, M. A. \& Terwilliger, T. C. (2006). Nat. Struct. Mol. Biol. 13, 184-185.

Grindon, C., Harris, S., Evans, T., Novik, K., Coveney, P. \& Laughton, C. (2004). Philos. Trans. R. Soc. Lond. A Math. Phys. Eng. Sci. 362, $1373-1386$.

Groom, C. R., Bruno, I. J., Lightfoot, M. P. \& Ward, S. C. (2016). Acta Cryst. B72, 171-179.

Gunsteren, W. F. van, Dolenc, J. \& Mark, A. E. (2008). Curr. Opin. Struct. Biol. 18, 149-153.

Hendrickson, W. A. \& Konnert, J. H. (1980). Computing in Crystallography, edited by R. Diamond, S. Ramaseshan \& K. Venkatesan, pp. 13.01-13.26. Bangalore: Indian Academy of Sciences.

Hintze, B. J., Lewis, S. M., Richardson, J. S. \& Richardson, D. C. (2016). Proteins, 84, 1177-1189.

Jack, A. \& Levitt, M. (1978). Acta Cryst. A34, 931-935.

Jain, S., Richardson, D. C. \& Richardson, J. S. (2015). Methods Enzymol. 558, 181-212.

Janowski, P. A., Cerutti, D. S., Holton, J. M. \& Case, D. A. (2013). J. Am. Chem. Soc. 135, 7938-7948.

Janowski, P. A., Liu, C., Deckman, J. \& Case, D. A. (2015). Protein Sci. 25, 87-102. 
Jorgensen, W. L., Chandrasekhar, J., Madura, J. D., Impey, R. W. \& Klein, M. L. (1983). J. Chem. Phys. 79, 926-935.

Joung, I. S. \& Cheatham, T. E. (2009). J. Phys. Chem. B, 113, 1327913290.

Liebschner, D., Afonine, P. V., Baker, M. L., Bunkóczi, G., Chen, V. B., Croll, T. I., Hintze, B., Hung, L.-W., Jain, S., McCoy, A. J., Moriarty, N. W., Oeffner, R. D., Poon, B. K., Prisant, M. G., Read, R. J., Richardson, J. S., Richardson, D. C., Sammito, M. D., Sobolev, O. V., Stockwell, D. H., Terwilliger, T. C., Urzhumtsev, A. G., Videau, L. L., Williams, C. J. \& Adams, P. D. (2019). Acta Cryst. D75, 861-877.

Liu, C., Janowski, P. A. \& Case, D. A. (2015). Biochim. Biophys. Acta, 1850, 1059-1071.

Lovell, S. C., Davis, I. W., Arendall, W. B. III, de Bakker, P. I. W., Word, J. M., Prisant, M. G., Richardson, J. S. \& Richardson, D. C. (2003). Proteins, 50, 437-450.

Maier, J. A., Martinez, C., Kasavajhala, K., Wickstrom, L., Hauser, K. E. \& Simmerling, C. (2015). J. Chem. Theory Comput. 11, 36963713.

Moriarty, N. W. (2015). Comput. Crystallogr. Newsl. 6, 26.

Moriarty, N. W., Grosse-Kunstleve, R. W. \& Adams, P. D. (2009). Acta Cryst. D65, 1074-1080.

Moriarty, N. W., Tronrud, D. E., Adams, P. D. \& Karplus, P. A. (2014). FEBS J. 281, 4061-4071.

Moriarty, N. W., Tronrud, D. E., Adams, P. D. \& Karplus, P. A. (2016). Acta Cryst. D72, 176-179.

Moulinier, L., Case, D. A. \& Simonson, T. (2003). Acta Cryst. D59, 2094-2103.

Murshudov, G. N., Skubák, P., Lebedev, A. A., Pannu, N. S., Steiner, R. A., Nicholls, R. A., Winn, M. D., Long, F. \& Vagin, A. A. (2011). Acta Cryst. D67, 355-367.

Murshudov, G. N., Vagin, A. A. \& Dodson, E. J. (1997). Acta Cryst. D53, 240-255.

Parkinson, G., Vojtechovsky, J., Clowney, L., Brünger, A. T. \& Berman, H. M. (1996). Acta Cryst. D52, 57-64.

Priestle, J. P. (2003). J. Appl. Cryst. 36, 34-42.

Richardson, D. C. \& Richardson, J. S. (2001). International Tables for Crystallography, Vol. F, edited by M. G. Rossmann \& E. Arnold, pp. 727-730. Dortrecht: Kluwer Academic Press.

Richardson, J. S. \& Richardson, D. C. (2018). Comput. Crystallogr. Newsl. 9, 21-24.

Richardson, J. S., Williams, C. J., Videau, L. L., Chen, V. B. \& Richardson, D. C. (2018). J. Struct. Biol. 204, 301-312.
Roe, D. R. \& Cheatham, T. E. (2013). J. Chem. Theory Comput. 9, 3084-3095.

Roitberg, A. \& Elber, R. (1991). J. Chem. Phys. 95, 9277-9287.

Schnieders, M. J., Fenn, T. D., Pande, V. S. \& Brunger, A. T. (2009). Acta Cryst. D65, 952-965.

Sheldrick, G. M. (2015). Acta Cryst. C71, 3-8.

Showalter, S. A. \& Brüschweiler, R. (2007). J. Chem. Theory Comput. 3, 961-975.

Simmerling, C., Fox, T. \& Kollman, P. A. (1998). J. Am. Chem. Soc. 120, 5771-5782.

Tahirov, T. H., Oki, H., Tsukihara, T., Ogasahara, K., Yutani, K., Ogata, K., Izu, Y., Tsunasawa, S. \& Kato, I. (1998). J. Mol. Biol. 284, 101-124.

Tickle, I. J., Laskowski, R. A. \& Moss, D. S. (2000). Acta Cryst. D56, 442-450.

Touw, W. G. \& Vriend, G. (2010). Acta Cryst. D66, 1341-1350.

Tronrud, D. E., Berkholz, D. S. \& Karplus, P. A. (2010). Acta Cryst. D66, 834-842.

Tronrud, D. E., Ten Eyck, L. F. \& Matthews, B. W. (1987). Acta Cryst. A43, 489-501.

Wall, M. E., Adams, P. D., Fraser, J. S. \& Sauter, N. K. (2014). Structure, 22, 182-184.

Wang, J., Wang, W., Kollman, P. A. \& Case, D. A. (2006). J. Mol. Graph. Model. 25, 247-260.

Wang, J., Wolf, R. M., Caldwell, J. W., Kollman, P. A. \& Case, D. A. (2004). J. Comput. Chem. 25, 1157-1174.

Waser, J. (1963). Acta Cryst. 16, 1091-1094.

Williams, C. J., Headd, J. J., Moriarty, N. W., Prisant, M. G., Videau, L. L., Deis, L. N., Verma, V., Keedy, D. A., Hintze, B. J., Chen, V. B., Jain, S., Lewis, S. M., Arendall, W. B. III, Snoeyink, J., Adams, P. D., Lovell, S. C., Richardson, J. S. \& Richardson, D. C. (2018). Protein Sci. 27, 293-315.

Williams, C. J., Videau, L. L., Hintze, B. J., Richardson, D. C. \& Richardson, J. S. (2018). bioRxiv, 324517.

Word, J. M., Lovell, S. C., LaBean, T. H., Taylor, H. C., Zalis, M. E., Presley, B. K., Richardson, J. S. \& Richardson, D. C. (1999). J. Mol. Biol. 285, 1711-1733.

Word, J. M., Lovell, S. C., Richardson, J. S. \& Richardson, D. C. (1999). J. Mol. Biol. 285, 1735-1747.

York, D. M., Darden, T. A. \& Pedersen, L. G. (1993). J. Chem. Phys. 99, 8345-8348.

Zagrovic, B., Gattin, Z., Lau, J. K.-C., Huber, M. \& van Gunsteren, W. F. (2008). Eur. Biophys. J. 37, 903-912. 